\title{
Élaboration d'indicateurs de blessures chez les enfants et les jeunes des Premières nations et inuits au Canada grâce à une méthode de Delphes modifiée
}

\author{
I. Pike, Ph. D. (1, 2, 3); R. J. McDonald, Ph. D. (3, 4); S. Piedt, B.A. (2, 3); A. K. Macpherson, Ph. D. (3, 5)
}

Cet article a fait l'objet d'une évaluation par les pairs.

\section{Résumé}

Introduction : Le but de cette recherche était de franchir la première étape de l'élaboration d'indicateurs valides et représentatifs des blessures chez les enfants et les jeunes des Premières nations et Inuits du Canada.

Méthodologie : À l'aide d'une méthode de Delphes modifiée, des intervenants des collectivités et des experts ont évalué chaque indicateur en fonction de son utilité perçue et de sa capacité à inciter à l'action dans le but de réduire les blessures chez les enfants et les jeunes des collectivités autochtones. Le processus s'est déroulé en 5 étapes et a permis d'obtenir un ensemble de 27 indicateurs.

Résultats : Les indicateurs évalués comme étant les plus utiles et les plus susceptibles d'inciter à l'action ont été ceux liés au taux de collision de véhicules à moteur, au taux de mortalité et au taux d'hospitalisation. Ont été sélectionnés ensuite les indicateurs de la formation en prévention des blessures et programmes communautaires d'intervention, ceux des blessures intentionnelles, ceux des brûlures et des chutes et enfin ceux du suicide.

Conclusion : Les résultats indiquent qu'utiliser une méthode de Delphes modifiée avec participation élargie est efficace et pertinent pour élaborer un ensemble d'indicateurs visant à guider les activités de prévention des blessures chez les enfants et les jeunes des Premières nations et Inuits, tout en respectant les principes $\mathrm{PCAP}^{\mathrm{MC}}$ (propriété, contrôle, accès et possession). Une fois achevés les travaux complémentaires de validation des indicateurs et la collecte des données associées, ces indicateurs vont pouvoir servir aux collectivités pour la surveillance des blessures et pour la prise de décisions et de mesures efficaces de réduction des blessures chez les enfants et les jeunes.

Mots-clés : Premières nations, Inuits, Autochtones, indicateurs de blessures, technique de Delphes modifiée, surveillance
Diffuser cet article sur Twitter

\section{Introduction}

Les blessures sont considérées comme un grave problème de santé, qui frappe particulièrement les personnes les plus vulnérables : les enfants, les jeunes, les personnes âgées et les Autochtones ${ }^{1}$. Elles sont la principale cause de mortalité chez les enfants, les jeunes et les jeunes adultes canadiens - une situation particulièrement préoccupante pour les membres des Premières nations et pour les collectivités inuites, car plus de $50 \%$ de ces populations autochtones sont âgées de moins de 25 ans.

Les blessures sont de loin la plus grande source d'années potentielles de vie perdues (APVP) pour les membres des Premières nations*. Les blessures sont à l'origine de $26 \%$ des décès chez les membres des Premières nations, contre $6 \%$ de l'ensemble des décès au Canada ${ }^{2,3}$, soit environ 3,5 fois la moyenne nationale. Le taux de blessures chez les adolescents autochtones est presque 4 fois plus élevé que celui des Canadiens non autochtones, et les garçons et les filles des Premières nations sont respectivement 5 fois et 7 fois plus nombreux à mourir par suicide que les jeunes des autres groupes ${ }^{1,4}$. Les taux d'hospitalisation attribuable à une blessure sont aussi significativement plus élevés

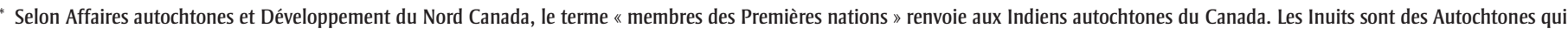
vivent surtout au Nunavut, dans les Territoires du Nord-Ouest, dans le nord du Québec et dans le nord du Labrador.

Rattachement des auteurs :

1. Département de pédiatrie, Faculté de médecine, Université de la Colombie-Britannique, Vancouver (Colombie-Britannique), Canada

2. B.C. Injury Research and Prevention Unit, Institut de recherche sur l'enfant et la famille, Hôpital pour enfants de la C.-B., Vancouver (Colombie-Britannique), Canada

3. Groupe de travail sur les indicateurs de blessures chez les enfants et les jeunes des Premières nations et Inuits*

4. Services de recherche et de gestion Katenies, territoire mohawk d'Akwesasne, Cornwall (Ontario), Canada

5. École de kinésiologie et des sciences de la santé, Faculté de la santé, Université York, Toronto (Ontario), Canada

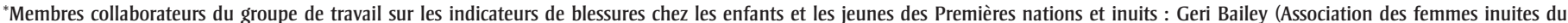

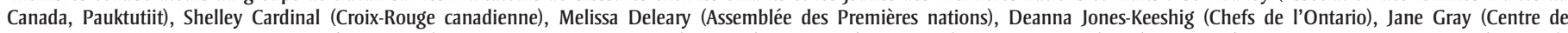

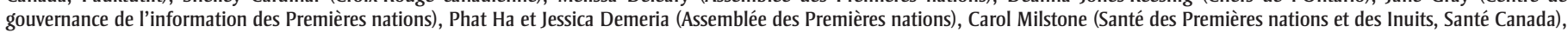
Looee Okalik (Inuit Tapiriit Kanatami), Heather Tait (Statistique Canada) et Parminder Thiara (Santé des Premières nations et des Inuits, Santé Canada).

Correspondance : Shannon Piedt, B.C. Injury Research and Prevention Unit, F508-4480 Oak Street, Vancouver (Colombie-Britannique) V6H 3V4; tél. : 604-875-2000, poste 5478; téléc. : 604-875-3569; courriel : spiedt@cw.bc.ca
} 
(deux fois plus) chez les enfants et les jeunes des régions où la proportion de résidents autochtones est élevée que chez ceux des régions où elle est faible ${ }^{5}$.

Pour réduire ces disparités en matière de blessures, on recommande des approches respectueuses qui soient concertées, durables et adaptées à la culture et qui tiennent compte des identités spécifiques des peuples des Premières nations et des Inuits $^{2,6}$. En 2004, la Coalition canadienne de la santé des enfants et des jeunes a placé la prévention des blessures et des traumatismes dans la liste des secteurs pour lesquels il convenait de créer des indicateurs de santé des nourrissons, des enfants et des jeunes au Canada ${ }^{7}$. En dépit de cela, le Canada accuse un retard par rapport à d'autres pays équivalents dans l'élaboration de plusieurs indicateurs importants portant sur la santé des enfants et des jeunes ${ }^{8}$. Un plan stratégique de prévention sur cinq ans a révélé la nécessité de définir des stratégies et des programmes de prévention des blessures dans les collectivités inuites et de mettre sur pied un réseau intégré de surveillance pour mesurer les tendances en matière de blessures ${ }^{9}$. Par ailleurs, bien que l'Enquête régionale longitudinale sur la santé des Premières nations permette de recueillir des données précieuses sur les individus et sur les collectivités, dont certaines portent sur les blessures, il n'existe actuellement aucune collecte systématique de données d'ensemble sur les blessures des enfants et des jeunes des Premières nations à l'échelle du Canada.

Le but de cette recherche était de procéder à la première étape de l'élaboration d'indicateurs valides représentatifs des blessures chez les enfants et les jeunes des Premières nations et Inuits au Canada. Nous nous sommes appuyés sur les travaux préliminaires de l'Équipe de développement des indicateurs de blessure au Canada, un groupe national de chercheurs, de praticiens et de décideurs travaillant sur la prévention des blessures, qui a sélectionné des indicateurs nationaux de blessures chez les enfants et les jeunes au Canada ${ }^{10}$. Selon Cryer ${ }^{11, p .3-1}$, un indicateur de blessures est [traduction] " une mesure sommaire qui représente, directement ou indirectement, les varia- tions et les tendances en matière de blessures ou de phénomènes liés aux blessures ou à la prévention de ces dernières ou en est révélatrice ". Plus spécifiquement, les objectifs de l'étude étaient de créer un groupe de travail solide constitué de personnes et d'organismes représentant les Autochtones et de définir et d'élaborer un ensemble d'indicateurs valides susceptible de servir de référence aux collectivités inuites et aux Premières nations pour documenter, analyser et communiquer des données sur les blessures chez les enfants et les jeunes. Une fois les données associées aux indicateurs recueillies, l'information va pouvoir servir à éclairer la prise de décision et la planification des mesures en matière de prévention des blessures dans les collectivités. Le suivi au fil du temps de ces indicateurs permettra de documenter la manière dont évolue le profil d'un groupe ou d'une collectivité en termes de blessures $^{12}$.

Un indicateur est valide lorsqu'il mesure réellement ce qu'il est censé mesurer ${ }^{13}$. Le choix des indicateurs dans cette étude repose sur les travaux de l'Effort international de collaboration sur les statistiques relatives aux blessures ${ }^{11}$ de 2001 et sur les travaux ultérieurs de Cryer et de ses collègues ${ }^{14}$, qui ont déterminé quels étaient les critères de validité d'un indicateur. Il en ressort qu'un indicateur idéal portant sur les blessures devrait :

- présenter une définition de cas s’appuyant sur un diagnostic (de lésion anatomique ou physiologique);

- être axé sur les blessures graves;

- être, dans la mesure du possible, associé à une vérification de cas impartiale;

- relever de données représentatives de la population cible;

- dépendre de systèmes de données existants (ou il devrait être facile d'élaborer de nouveaux systèmes de données pour l'alimenter);

- posséder une définition écrite complète.

\section{Méthodologie}

Début 2007, la Direction générale de la santé des Premières nations et des Inuits de Santé Canada a convié l’Équipe de développement des indicateurs de bles- sures au Canada à monter un projet de 3 ans visant l'établissement d'indicateurs de blessures chez les enfants et les jeunes des Premières nations et Inuits. Au Canada, les peuples des Premières nations et les Inuits sont représentés par de nombreux organismes autochtones locaux, régionaux et nationaux, de même que par les ministères fédéraux responsables de la prestation des programmes de santé et des programmes sociaux, ce qui inclut les mesures de réduction des blessures.

Le processus et la méthodologie de ce projet visaient dès la conception à assurer un équilibre entre la rigueur scientifique et une approche axée sur la collectivité respectant les principes $\mathrm{PCAP}^{\mathrm{MC}}$ (propriété, contrôle, accès et possession) qui s'appliquent à la collecte de données sur les peuples autochtones au Canada. Cela signifie que la propriété, le contrôle, l'accès et la possession des données relèvent de la communauté autochtone ${ }^{15}$. Le processus devait respecter une approche pratique dans le développement des indicateurs.

C’est la Direction générale de la santé des Premières nations et des Inuits de Santé Canada qui a sélectionné les participants à cette recherche et elle a inclus pour ce faire des représentants de l'Assemblée des Premières nations, de l'Inuit Tapiriit Kanatami, de la Gendarmerie royale du Canada, du ministère des Affaires autochtones et Développement du Nord Canada, de la fondation Sauve-qui-pense, du Centre hospitalier pour enfants de l'est de l'Ontario, du programme Plan-It-Safe, des services de recherche et de gestion Katenies, de Statistique Canada, du ministère de la Santé et du Développement social du Nunatsiavut et de l'Association des femmes inuites du Canada (Pauktutiit). Vingt et un participants provenant de ces organismes se sont réunis pour la planification du projet et ont entamé le processus, constituant ainsi le Groupe de travail sur le projet des indicateurs de blessures chez les enfants et les jeunes des Premières nations et Inuits.

Nous avons adapté la méthode de Delphes multiphasique modifiée à partir des méthodes décrites par Lindsay et ses collègues ${ }^{16}$ et nous l'avons appliquée à l'élaboration 
des indicateurs de blessures chez les enfants et les jeunes des Premières nations et Inuits. Le choix des indicateurs a reposé sur des données accessibles limitées traitant du fardeau des blessures chez les enfants et les jeunes des Premières nations et Inuits, sur des recherches et des pratiques exemplaires antérieures à propos de la prévention et enfin sur la rétroaction des experts du Groupe de travail et de leurs réseaux respectifs.

\section{Phase I : revue de la littérature}

La phase I constituait en une revue de la littérature pertinente avec l'objectif de repérer tous les indicateurs valides fondés sur des données probantes portant sur les blessures chez les enfants et les jeunes des Premières nations et Inuits. Les analystes de la Direction générale de la santé des Premières nations et des Inuits de Santé Canada ont réalisé cette revue de la littérature en utilisant la méthodologie de Pike et ses collègues ${ }^{10}$ et en utilisant les bases de données suivantes pour la période 1985-2007 : Medline, Ovid, Transport, Transportation Research Information Services, Sportdiscus, Cumulative Index to Nursing and Allied Health Literature, Embase, Psychinfo, Healthstar et Hispanic American Periodicals Index. Ils ont également fait des recherches sur les sites Web d'organismes autochtones et du gouvernement et sur des listes de rapports de programmes afin d'avoir accès à la littérature grise pertinente. Ils ont relevé et résumé 10 études au sein des publications évaluées par des pairs et de la littérature grise (liste fournie sur demande par les auteurs). Cette revue de la littérature a fourni une première liste de 48 indicateurs.

\section{Phase II : élaboration des principales catégories de blessures et classification des indicateurs}

Sur les 21 membres du Groupe de travail, 19 ont pu se réunir et se sont mis d'accord sur 4 catégories permettant de classer les indicateurs de blessures chez les enfants et les jeunes des Premières nations et Inuits : sécurité en milieu de travail, à la maison et dans les lieux publics; transports; sports et loisirs et blessures intentionnelles (autoinfligées ou liées à un acte de violence [« blessures violentes »]). Se fondant sur leur expertise, sur leur expérience personnelle et sur leurs connaissances du sujet, les membres du groupe ont discuté des blessures les plus fréquentes à inscrire dans chaque catégorie et de la manière d'effectuer la mesure et la surveillance de ces blessures. Ils se sont mis d'accord sur 4 types d'indicateurs : ceux portant sur les résultats, sur les facteurs de risque et les facteurs de protection, sur les programmes et sur les politiques.

Par la suite, le groupe s'est scindé en 4 sous-groupes, un par catégorie de blessures, et a travaillé sur les 48 indicateurs issus de la revue de littérature, avec la possibilité d'ajouter d'autres indicateurs au besoin. Après analyses et discussions, chaque sous-groupe a présenté sa liste d'indicateurs à l'ensemble du groupe. Au total, 170 indicateurs ont été sélectionnés.

Pour réduire le nombre d'indicateurs tout en conservant ceux considérés comme les plus importants et représentatifs des enjeux des collectivités portant sur les blessures chez les enfants et les jeunes, le Groupe de travail a entrepris de hiérarchiser les indicateurs. Pour réaliser cette tâche, on a affiché la liste des indicateurs sur des tableaux de présentation. Chaque participant disposait de 55 pastilles adhésives (correspondant à environ le tiers du nombre d'indicateurs affichés) à placer à côté des indicateurs qu'il considérait comme les plus importants. Tous les indicateurs ayant reçu au moins 10 pastilles (ce qui fournissait une première indication de l'importance de l'indicateur) ont été retenus et les autres ont été rejetés. Grâce à cette technique de priorisation du tiers $(\mathrm{N} / 3)^{17}$, on a obtenu 62 indicateurs, qui ont été regroupés par les participants en 7 (et non plus 4) grandes catégories : tous les types de blessures; morsures d'animaux et hypothermie ou engelure; blessures intentionnelles; brûlures et chutes; noyade; suicide et enfin collisions de véhicules à moteur.

L'importance de chaque indicateur a été déterminée par deux critères : l'indicateur devait représenter un lourd fardeau pour les Premières nations et les Inuits, leurs familles et le système de santé et il devait pouvoir être l'objet de mesures de prévention. Les participants avaient en outre accès aux critères de validité des indicateurs de l'Effort de collaboration internationale sur les indicateurs de blessures (ICEIInG) pour éclairer leur prise de décision.

À l'étape suivante, la liste des 62 indicateurs a été revue et retravaillée. On a demandé à chaque membre du Groupe de travail de consulter son sous-groupe et de recommander, pour chaque indicateur, soit son maintien, soit son élimination, soit d'indiquer une incertitude à son propos, en s'appuyant sur 3 questions : 1) cet indicateur est-il important dans ma collectivité? 2) Cet indicateur m'aiderait-il à surveiller les blessures dans ma collectivité? 3) Cet indicateur fournit-il une information suffisante pour agir en prévention des blessures chez les enfants et les jeunes de ma collectivité?

Nous avons analysé les réponses et avons retenu les indicateurs qu'une majorité des membres de groupe de travail avait recommandé de conserver. Ceux qu'une majorité avait recommandé d'éliminer ont été éliminés. (Aucun indicateur n’a reçu un vote majoritaire d'incertitude.) $\mathrm{Au}$ cours de cette phase et à la suite de discussions, les membres du Groupe de travail ont proposé 2 autres indicateurs, qui ont été évalués et jugés suffisamment importants pour être conservés. Il s'agit du pourcentage d'enfants et de jeunes inscrits dans des programmes d'apprentissage de la natation et du pourcentage de délinquants violents participant à des programmes de justice réparatrice, deux indicateurs qui étaient dans la liste des indicateurs potentiels. À cette étape, la liste comportait 36 indicateurs.

\section{Phase III : rétroaction des régions}

Nous avons recueilli les avis d'utilisateurs potentiels à l'échelle des collectivités. Les chercheurs ont assisté à des réunions régionales et ont recruté des praticiens et des décideurs en matière de prévention des blessures chez les Premières nations et les Inuits. À chaque réunion, on a expliqué le projet et on a demandé aux participants de donner leur avis sur la liste des 36 indicateurs de blessures chez les enfants et les jeunes. 
Nous avons obtenu, pour chaque indicateur, les avis d'un certain nombre d'organismes régionaux de l'Alberta, du Manitoba, de l'Ontario, du Québec et du Nunatsiavut : ceux du Groupe de travail sur le bien-être au sein des communautés du Manitoba, du Groupe de travail régional sur la prévention des blessures de l'Assemblée des Premières nations, du Cercle de la petite enfance des Premières nations (représentants de l'Initiative d'aide préscolaire aux Autochtones de la Saskatchewan et de la Fédération des nations indiennes de la Saskatchewan), des Chefs de l'Ontario et du Conseil national inuit sur la santé.

À cette étape du processus, les représentants des organismes régionaux ont sélectionné 7 autres indicateurs jugés importants pour comprendre et prévenir les blessures chez les enfants et les jeunes au sein de leurs collectivités. La liste est donc passée de 36 à 43 indicateurs.

\section{Phase IV : spécifications des indicateurs}

En nous basant sur les modèles de rapports déjà publiés en Australie ${ }^{18}$, en NouvelleZélande $^{19}$, en Europe $^{20}$ et au Canada ${ }^{21}$, nous avons créé un modèle de spécification pour les indicateurs (tableau 1) et nous

TABLEAU 1

Modèle global de spécification des indicateurs de blessures chez les enfants et les jeunes

\begin{tabular}{l}
\multicolumn{1}{c}{ Indicateur } \\
\hline Définition de l'indicateur \\
Définition des termes pertinents associés \\
Justification du choix de cet indicateur \\
Définition de cas opérationnelle \\
Méthode de calcul \\
Numérateur \\
Dénominateur \\
Sources des données, accessibilité et qualité, \\
pour les années sélectionnées \\
Unités de mesure \\
Guide d'utilisation \\
Champ d'utilisation de l'indicateur \\
Spécifications des données \\
Restrictions \\
Comment utiliser cet indicateur \\
\hline
\end{tabular}

avons rédigé une version préliminaire des spécifications pour chacun des 43 indicateurs. Le Groupe de travail s'est ensuite réuni pour en discuter, pour revoir et pour retravailler les indicateurs et leurs spécifications, puis un autre cycle d'évaluation et de rétroaction a eu lieu par courriel. Neuf membres du groupe de travail ont accepté ${ }^{\dagger}$ d'évaluer encore une fois les indicateurs et ont recommandé d'éliminer plusieurs indicateurs en raison de lacunes dans les données disponibles et des difficultés et des coûts liés à la production de nouveaux systèmes de collecte de données en lien avec ces indicateurs. À la fin de la phase IV, on a donc abouti à une liste plus réduite, de 33 indicateurs (tableau 2).

\section{Phase $V$ : finalisation des indicateurs}

Après la phase de spécification des 33 indicateurs, le Groupe de travail s'est réuni une dernière fois en décembre 2008, 13 membres ayant assisté à la réunion. Ils ont évalué, pour chaque indicateur, son utilité et sa capacité à inciter à l'action pour réduire les blessures chez les enfants et les jeunes des Premières nations et inuits, à l'aide d'une échelle à 9 points (1 qualifiant une utilité ou une capacité à inciter à l'action faibles et 9 une grande utilité ou une forte capacité à inciter à l'action). Sept indicateurs ont été jugés inutiles et sans capacité d'inciter à l'action (et, par conséquent, ne satisfaisant pas les critères de validité), en raison d'un manque soit de données soit de ressources, et ils ont été de ce fait éliminés (voir les cellules ombrées dans le tableau 2). Le processus s'est terminé par l'adoption par le Groupe de travail d'une liste définitive de 27 indicateurs de blessures chez les enfants et les jeunes des Premières nations et inuits.

Après le vote, le groupe a accepté à l'unanimité de réintégrer l'indicateur des années potentielles de vie perdues (APVP) en raison d'une blessure (indicateur qui faisait partie de la liste des indicateurs potentiels à l'étape de la revue de littérature), et ce, bien que cet indicateur n'ait pas été soumis au processus d'évaluation.

\section{Résultats}

L'utilisation d'une méthode de Delphes modifiée a abouti à la création d'une liste de 27 indicateurs de blessures. Les indicateurs classés comme les plus utiles et les plus susceptibles d'inciter à l'action sont ceux liés aux collisions de véhicules à moteur, aux taux de mortalité et au nombre d'enfants et de jeunes hospitalisés pour tous les types de blessures. Viennent ensuite les indicateurs liés à la formation en prévention des blessures et aux programmes communautaires d'intervention, ceux liés aux blessures intentionnelles, aux brûlures et aux chutes et enfin au suicide, et ce, même si certains d'entre eux ont été évalués comme n'incitant guère à l'action.

\section{Analyse}

La méthode de Delphes modifiée que nous avons utilisée a permis de clore la première étape du processus d'élaboration des indicateurs avec l'obtention d'un ensemble de 27 indicateurs de blessures chez les enfants et les jeunes des Premières nations et Inuits qui est utilisable pour la prise de mesures de prévention des blessures visant les Autochtones du Canada. Malgré l'existence de variations entre experts dans l'évaluation de l'utilité et de la capacité d'incitation à l'action de chacun des indicateurs, un consensus d'ensemble s'est dégagé. Les scores élevés obtenus par certains indicateurs indiquent qu'ils répondent aux besoins de ceux qui travaillent à prévenir les blessures chez les enfants et les jeunes des Premières nations et inuits.

Les indicateurs ciblaient les enfants et les jeunes des Premières nations et inuits, mais certains sont applicables à tous les enfants et jeunes vivant en milieu rural ou en région éloignée, et d'autres sont applicables aux enfants et aux jeunes en général.

\section{Points forts et limites}

Ce travail comporte certaines limites, qu'il est important de souligner ici.

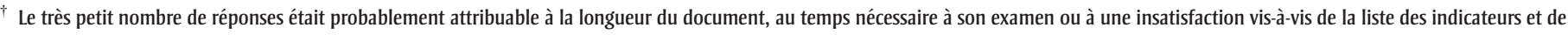
leurs spécifications.
} 
TABLEAU 2

Évaluation de l'utilité de chaque indicateur et de sa capacité à inciter à l'action dans la prévention des blessures chez les enfants et les jeunes des Premières nations et Inuits

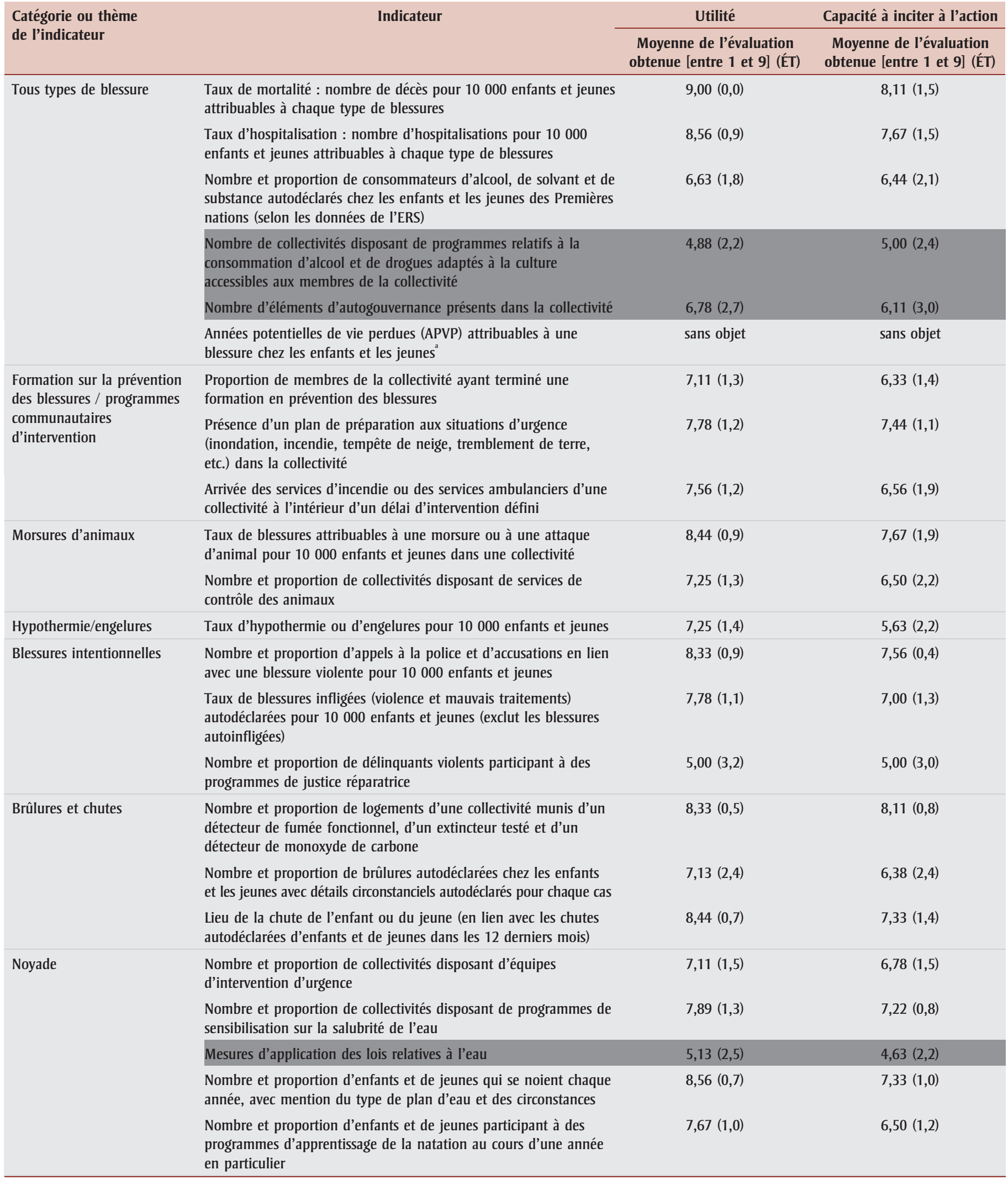


TABLEAU 2 (Suite)

Évaluation de l'utilité de chaque indicateur et de sa capacité à inciter à l'action dans la prévention des blessures chez les enfants et les jeunes des Premières nations et Inuits

\begin{tabular}{|c|c|c|c|}
\hline \multirow{2}{*}{$\begin{array}{l}\text { Catégorie ou thème } \\
\text { de l'indicateur }\end{array}$} & \multirow[t]{2}{*}{ Indicateur } & Utilité & \multirow{2}{*}{$\begin{array}{l}\text { Capacité à inciter à l'action } \\
\text { Moyenne de l'évaluation } \\
\text { obtenue [entre } 1 \text { et 9] (ÉT) }\end{array}$} \\
\hline & & $\begin{array}{l}\text { Moyenne de l'évaluation } \\
\text { obtenue [entre } 1 \text { et } 9 \text { ] (ÉT) }\end{array}$ & \\
\hline \multirow[t]{3}{*}{ Suicide } & $\begin{array}{l}\text { Nombre de collectivités disposant de programmes de promotion du } \\
\text { bien-être et de programmes de santé mentale }\end{array}$ & $6,50(2,8)$ & $6,86(2,3)$ \\
\hline & $\begin{array}{l}\text { Taux de problèmes de santé mentale autodéclarés chez les enfants } \\
\text { et les jeunes }\end{array}$ & $7,89(0,8)$ & $6,56(1,9)$ \\
\hline & $\begin{array}{l}\text { Taux d'appels à un centre d'intervention téléphonique en prévention } \\
\text { du suicide, par zone géographique }\end{array}$ & $7,67(1,0)$ & $7,22(0,8)$ \\
\hline \multirow[t]{4}{*}{$\begin{array}{l}\text { Collisions de } \\
\text { véhicules à moteur }\end{array}$} & $\begin{array}{l}\text { Taux de collisions de véhicules à moteur impliquant un enfant ou un } \\
\text { jeune, par type de véhicule et avec mention des circonstances de } \\
\text { l'accident }\end{array}$ & $8,78(0,4)$ & $8,00(1,0)$ \\
\hline & $\begin{array}{l}\text { Proportion de véhicules à moteur équipés d'un dispositif de retenue } \\
\text { pour enfant (siège d'auto pour enfant) ou d'un siège d'appoint } \\
\text { adéquat, par collectivité }\end{array}$ & $8,78(0,4)$ & $8,33(1,0)$ \\
\hline & $\begin{array}{l}\text { Âge et sexe du conducteur et des passagers impliqués dans un } \\
\text { accident de la route par type de véhicule (voiture, fourgonnette, } \\
\text { camion, VTT, motoneige) et par type d'usager de la route } \\
\text { (conducteur, passager, piéton, cycliste) }\end{array}$ & $8,33(0,9)$ & $7,67(1,4)$ \\
\hline & $\begin{array}{l}\text { Présence d'une loi sur l'âge minimum pour conduire un VTT. } \\
\text { Nombre de provinces et de territoires disposant d'une loi sur l'âge } \\
\text { minimum pour conduire un VTT. }\end{array}$ & $7,13(2,2)$ & $6,00(2,7)$ \\
\hline
\end{tabular}

Abréviations : APVP, Années potentielles de vie perdues; ERS, Enquête régionale longitudinale sur la santé des Premières nations; ÉT, écart-type; VTT, véhicule tout-terrain.

Remarque : Les zones ombrées représentent les indicateurs ayant été faiblement évalués et ayant été éliminés par la suite.

a Les membres du groupe d'experts ont accepté à l'unanimité d'inclure les APVP comme indicateur utile, mais cet indicateur n'a pas été évalué.

En premier lieu, la littérature sur la prévention des blessures chez les enfants et les jeunes autochtones permettant d'éclairer la prise de décision quant à la sélection des indicateurs est très pauvre.

La méthode de Delphes modifiée que nous avons utilisée est subjective et repose sur l'expertise et l'expérience des participants. Malgré les tentatives d'objectivité dans le choix et dans la hiérarchisation des indicateurs aux différentes étapes du processus, les résultats demeurent dépendants de l'opinion des experts participants. On leur a fourni des critères de validité d'un indicateur, mais on ignore dans quelle mesure ces critères ont influencé leur choix. Les résultats seraient donc probablement différents avec un groupe d'experts différent. Cependant, les experts que nous avons sélectionnés étaient selon nous les plus aptes à participer au processus, à la fois car ils connaissaient bien le domaine et car ils étaient représentatifs de leur organisme et de ses membres.

Une autre limite est le manque de données pour alimenter les indicateurs. Pour plusieurs indicateurs, aucune donnée n'était disponible, lacune qui risque de ne pas être comblée dans un avenir proche. Il demeure que les données associées à bon nombre d'indicateurs sont déjà disponibles grâce à l'Enquête longitudinale régionale sur la santé des Premières nations, et que certaines collectivités (en particulier les 10 bandes de la nation Secwepemc en Colombie-Britannique) ont recueilli des données sur la santé et les blessures susceptibles d'alimenter les indicateurs choisis. De plus, nous prévoyons que, progressivement, de plus en plus de collectivités recueilleront les données d'intérêt local qui leur sont utiles pour prévenir les blessures chez les enfants et les jeunes, ce qui est une approche conforme aux principes $\mathrm{PCAP}^{\mathrm{MC}} 15$.

\section{Conclusion}

L'utilisation d'une méthode de Delphes modifiée interdisciplinaire, qui a requis la participation directe des experts des 
Premières nations et des Inuits et un bon leadership de leur part, a abouti à la création d'une liste de 27 indicateurs utiles et incitant à l'action, dont le but est de guider les mesures de prévention des blessures chez les enfants et les jeunes des Premières nations et inuits.

Quoique plusieurs indicateurs soient similaires à ceux utilisés pour les enfants et les jeunes non autochtones du Canada ${ }^{10}$, nous avons relevé plusieurs différences. Ce qui est le plus important, c'est que les indicateurs soient caractéristiques des blessures chez les enfants et les jeunes des Premières nations et Inuits et qu'ils reflètent les circonstances et les conditions locales ayant une incidence sur la prévention des blessures et la gestion des risques liés à ces dernières dans les collectivités autochtones, dont certaines sont petites, en milieu rural ou en zone éloignée. Par exemple, les indicateurs des Premières nations et des Inuits portent sur la formation en prévention des blessures et les programmes communautaires d'intervention, sur les morsures d'animaux, sur la noyade, sur l'hypothermie et les engelures, éléments qui sont considérés comme moins importants au sein de la population non autochtone.

Les recherches et les collaborations à venir entre le Groupe de travail et les collectivités autochtones vont permettre de mesurer l'utilité des indicateurs en matière de prévention des blessures. Nous devons sélectionner l'information et les données pertinentes pour alimenter les indicateurs. Il est prévu que l'équipe de recherche collabore avec les collectivités pour cela, ce qui implique notamment de mettre au point diverses définitions portant sur les causes et sur la gravité des blessures. Les autorités sanitaires et les collectivités autochtones pourront alors utiliser les données pour planifier, réaliser et évaluer des programmes et des mesures de prévention des blessures chez les enfants et les jeunes, ce qui est conforme aux principes $\mathrm{PCAP}^{\mathrm{MC}}$ qui sous-tendent les travaux de recherche réalisés au sein des collectivités autochtones du Canada.

\section{Remerciements}

Cette étude a été financée par la Direction générale de la santé des Premières nations et des Inuits (DGSPNI) de Santé Canada, le Réseau de la recherche en santé chez les enfants et les jeunes de ColombieBritannique (CYHRNet) et les Instituts de recherche en santé du Canada (IRSC) (KTB-109190).

Nous tenons à remercier tous les membres du Groupe de travail sur les indicateurs de blessures chez les enfants et les jeunes des Premières nations et Inuits. Nous désirons remercier également la Direction générale de la santé des Premières nations et des Inuits (DGSPNI) de Santé Canada et le Réseau de la recherche en santé chez les enfants et les jeunes de ColombieBritannique (CYHRNet) pour avoir financé cette étude et pour avoir fourni dans les temps un accès aux ressources de base. Nous souhaitons remercier en outre l'Assemblée des Premières nations (APN), l'Inuit Tapiriit Kanatami (ITK), l'Association des femmes inuites du Canada (Pauktutiit) et les IRSC. Nous sommes très reconnaissants envers ces organismes pour les importantes ressources qu'ils ont mises à notre disposition et pour le soutien permanent dont nous avons bénéficié, ce qui a facilité la finalisation de la liste définitive des indicateurs. Nous voulons enfin remercier le Groupe de travail sur le bien-être au sein des communautés du Manitoba, l'APN, le Groupe de travail régional sur la prévention des blessures chez les Premières nations, le Cercle de la petite enfance des Premières nations (représentants l'Initiative d'aide préscolaire aux Autochtones de la Saskatchewan et la Fédération des nations indiennes de la Saskatchewan), les Chefs de l'Ontario, le Conseil des agents de la santé de l'APN, les représentants de l'Enquête régionale longitudinale sur la santé des Premières nations et le Comité inuit national de la santé pour leur contribution à l'établissement des indicateurs et au perfectionnement de la liste au cours du processus.

\section{Références}

1. Sauve-qui-pense. Mettre un terme à l'épidémie invisible du Canada : une stratégie pour la prévention des blessures. Toronto (Ont.) : Sauve-qui-pense; 2005.
2. Banerji A; Société canadienne de pédiatrie; Comité de la santé des Premières nations, des Inuits et des Métis. La prévention des blessures non intentionnelles chez les enfants et les adolescents autochtones au Canada. Paediatr Child Health. 2012;17(7):393-394.

3. Santé Canada. Profil statistique de la santé des Premières nations au Canada : utilisation des services de santé dans l'Ouest canadien, 2000 [Internet]. Ottawa (Ont.) : Santé Canada; 2009 [consulté le 15 avril 2012]. Consultable à partir de la page : http://www.hc-sc.gc.ca/fniah-spnia/pubs /aborig-autoch/2009-stats-profil-vol2/index -fra.php

4. Harrop AR, Brant RF, Ghali WA, Macarthur C. Injury mortality rates in Native and nonNative children : a population-based study. Public Health Rep. 2007;122(3):339-46.

5. Oliver LN, Kohen DE. Hospitalisations d'enfants et de jeunes pour blessure non intentionnelle dans les régions ayant un fort pourcentage de résidents d'identité autochtone, 2001-2002 à 2005-2006. Rapports sur la santé, 2012;23(3):7-16.

6. Anderson M, Smylie J, Anderson I, Sinclair R, Crengle S. First Nations, Métis, and Inuit health indicators in Canada : a background paper for the project "Action Oriented Indicators of Health and Health Systems Development for Indigenous Peoples in Australia, Canada, and New Zealand" [rapport de recherche]. 2006. PDF (167 Ko) téléchargeable à partir du lien : http:// www.med.uottawa.ca/SIM/data/Images /Aboriginal_health_indicators.pdf

7. Canadian Child and Youth Health Coalition (CCYHC). Our work : health indicators : child and youth health indicator program [Internet]. London (Ont) : CCYHC; 2008 [consulté le 12 avril 2013]. Consultable à la page : http://www.ccyhc.org/work_indicators_ about.html

8. Leitch K. Vers de nouveaux sommets : rapport de la conseillère en santé des enfants et des jeunes. Ottawa (Ont.) : Gouvernement du Canada; 2007.

9. Pauktuutit. Inuit Five-Year Injury Prevention Strategic Plan 2010-1015. Ottawa (Ont.) : Pauktuutit Inuit Women of Canada; 2010. 
10. Pike I, Piedt S, Warda $L$ et collab. Developing injury indicators for Canadian children and youth : a modified-Delphi approach. Inj Prev. 2010;16:154-60.

11. Cryer C. ICE Injury Indicators Group (ICEIInG) - progress report, aspirations, goals and strategy development. Dans : International Collaborative Effort on Injury Statistics, vol. IV. Washington (DC) : NCHS, CDC, US Department of Health and Human Services; 2003. PDF (3,22 Mo) téléchargeable à partir du lien : http://www .cdc.gov/nchs/data/ice/iceproceedings.pdf

12. Centre des Premières nations. Compréhension des indicateurs de santé. Ottawa (Ont.) : Organisation nationale de la santé autochtone; 2007.

13. Guilford JP, Fruchter D. Fundamental statistics in psychology and education (5 éd.). New York : McGraw-Hill; 1973.

14. Cryer C, Langley JD, Jarvis SN, Mackenzie SG, Stephenson SC, Heywood P. Injury outcome indicators : the development of a validation tool. Inj Prev. 2005;11:53-57.

15. Centre de gouvernance de l'information des Premières nations (CGIPN). Les principes PCAP des Premières nations [Internet]. Akwesasne (Ont.) : CGIPN, 2013 [consulté le 19 août 2013]. Consultable à la page : www.fnigc.ca/fr/pcap.html

16. Lindsay P, Schull M, Bronskill S, Anderson $\mathrm{G}$. The development of indicators to measure the quality of clinical care in emergency departments following a modifieddelphi approach. Acad Emerg Med. 2002;9: 1131-1139.

17. Andersen A. A process guidebook : tools and techniques for effective facilitation. Chicago (IL) : Arthur Andersen and Co; 1991.

18. Harrison JE, Steenkamp M. Technical review and documentation of current NHPA injury indicators and data sources. Adelaide (AU) : Australian Institute of Health and Welfare (AIHW); 2002. (catalogue AIHW n INJCAT 47, Injury Research and Statistics Series Number 14).
19. Cryer C, Langley J, Stephenson S. Developing valid injury outcome indicators: a report for the New Zealand Injury Prevention Strategy. Dunedin (NZ) : Injury Prevention Research Unit, Department of Preventive and Social Medicine, University of Otago; 2004.

20. Pencheon D. The good indicators guide : understanding how to choose and use indicators. Coventry (UK) : NHS Institute for Innovation and Improvement; 2008.

21. Institut canadien d'information sur la santé (ICIS). Indicateurs pancanadiens de soins de santé primaires, rapport 1 , volume 1 . Projet d'élaboration d'indicateurs pancanadiens de soins de santé primaires. Ottawa (Ont.) : ICIS; 2006. 